Projets

de paysage

\section{Projets de paysage}

Revue scientifique sur la conception et l'aménagement de l'espace

$10 \mid 2014$

Le paysage à l'épreuve de la transition énergétique

\title{
Communs paysagers et devenirs éoliens opposés
}

Le cas de la Seine-et-Marne (France)

Shared Landscapes and Opposition to Future Wind Farms - The Case of Seine-etMarne (France)

\section{Alain Nadaï et Olivier Labussière}

\section{OpenEdition}

\section{Journals}

Édition électronique

URL : http://journals.openedition.org/paysage/11668

DOI : 10.4000/paysage.11668

ISSN : 1969-6124

\section{Éditeur :}

École nationale supérieure du paysage de Versailles-Marseille, Institut national des sciences appliquées Centre Val de Loire - École de la nature et du paysage, École nationale supérieure d'architecture et de paysage de Bordeaux, École nationale supérieure d'architecture et de paysage de Lille, Agrocampus Angers

Référence électronique

Alain Nadaï et Olivier Labussière, " Communs paysagers et devenirs éoliens opposés », Projets de paysage [En ligne], 10 | 2014, mis en ligne le 31 août 2014, consulté le 15 octobre 2020. URL : http:// journals.openedition.org/paysage/11668; DOI : https://doi.org/10.4000/paysage.11668

Ce document a été généré automatiquement le 15 octobre 2020

Projets de paysage 


\title{
Communs paysagers et devenirs éoliens opposés
}

\author{
Le cas de la Seine-et-Marne (France) \\ Shared Landscapes and Opposition to Future Wind Farms - The Case of Seine-et- \\ Marne (France)
}

Alain Nadaï et Olivier Labussière

\section{NOTE DE L'AUTEUR}

Ce travail a été réalisé avec l'appui financier du conseil français de l'Énergie, du ministère de l'Écologie, du Développement durable et de la Mer (MEDDM - Programme PDD « Paysage et développement durable »), de l'Agence française de l'environnement et de la maîtrise de l'énergie (Ademe, convention nº 0710 C 0019), de la Région Île-deFrance (R2DS), du programme interdisciplinaire Énergie du CNRS et de l'Agence nationale de la recherche (ANR, programme sociétés innovantes, convention 2011SOIN-003-01, projet Collener).

1 Si le lien entre le paysage et l'éolien peut paraître évident du fait de leur dimension visuelle commune, la manière dont la dimension paysagère intervient dans les processus éoliens appelle, pour être comprise, une analyse circonstanciée. Cet article part d'un projet éolien contesté et conflictuel dans un village de Seine-et-Marne (France) et explore le rôle du paysage dans la montée des tensions autour de ce projet.

2 Ce dernier fut une première en Île-de-France. Il débuta en 2005, sa zone de développement éolienne fut adoptée en mai 2007, reçut un avis favorable de la préfecture en mai 2009, fut purgée de tout recours en 2012. Il devrait être en service à la fin 2014. Ce projet a suscité de violentes oppositions (rupture de liens familiaux, déstructuration de la vie associative, violences physiques, atteintes aux biens) et une désagrégation de l'articulation entre la sphère privée et la sphère publique, constitutive de la vie villageoise et de la pratique politique locale. Notre analyse suit 
l'émergence et la structuration de trajectoires d'opposants dans leur constitution progressive, à partir d'individus n'ayant pas a priori de position définie vis-à-vis de l'éolien.

3 Au cœur de ce drame éolien, nous explorons le rôle du " paysage d'État " au sens d'une tradition administrative de protection centralisée du paysage dans ses difficultés à prendre en charge des communs paysagers locaux pour soutenir l'émergence de projets éoliens partagés. La déstructuration de ces communs localement structurants laisse place à l'émergence de communs paysagers nés d'oppositions progressivement radicalisées à l'éolien.

4 Nous procédons en trois étapes. La première partie présente le contexte institutionnel de la politique éolienne française ainsi que notre méthode, en précisant notamment les notions de «paysage d'État» et de commun paysager. La seconde partie analyse le cas du village de Ventville ${ }^{1}$ (Seine-et-Marne, France) en mettant en avant la manière dont s'y joue la difficile articulation entre les dimensions publique et privée de la politique éolienne française. La troisième partie ouvre une discussion sur l'articulation entre paysage, communs et politique éolienne.

\section{Ressources et communs paysagers}

5 La politique éolienne française a émergé en 2000 avec l'adoption de tarifs d'achat de l'électricité éolienne et s'est déployée durant sept années sans vision d'aménagement du territoire (Nadaï, 2007a), jusqu'à la mise en œuvre des zones de développement éolien $(\mathrm{ZDE})^{2}$. Cette institutionnalisation a conduit à une privatisation de l'éolien en France, au travers d'un portage dominant des projets par les développeurs privés. Contrairement à ses homologues danoise et allemande, la ZDE à la française n'est pas un document d'urbanisme mais un contrat électrique conditionnant le bénéfice du tarif à l'implantation des éoliennes dans ces zones. Elle marque un mouvement de déconcentration plus que de décentralisation, l'approbation finale de ces zones relevant du préfet après propositions des communes ou des intercommunalités. Dans de rares cas, la ZDE trouve aussi une utilité en termes d'aménagement de l'espace et de coordination locale (Nadaï et Labussière, 2009).

\section{Paysage d'État et communs paysagers}

6 Le paysage intervient selon plusieurs statuts et modalités lors des processus qui soustendent l'autorisation des projets éoliens.

7 En amont des projets, lors de l'élaboration des ZDE et de leur validation par le préfet, ou encore lors de l'élaboration de schémas éoliens, les processus de planification territoriale croisent, à des degrés divers, des processus très codifiés (e.g. empilements cartographiques de contraintes administratives liées à la présence d'entités paysagères reconnues, de faune ou de flore protégées, d'éléments patrimoniaux inscrits ou classés) et des processus plus ouverts (e.g. consultations publiques, concertations). Ces derniers permettent de prendre en compte des pratiques paysagères peu codifiées ou des attachements locaux aux paysages. 
Lors des études d'impact et des avis administratifs sur les permis de construire qui fondent le plus souvent (avec les enquêtes publiques) les avis du préfet, les mêmes éléments peuvent intervenir.

Lors des enquêtes publiques, positionnées entre l'avis administratif et celui du préfet, les pétitionnaires sont censés pouvoir exprimer leur attachement à tels ou tels éléments de paysage, en évoquer les pratiques locales, quotidiennes ou encore pointer l'absence (dans les études d'impact ou les avis administratifs) de prise en compte d'éléments de paysages, de faune ou de flore protégés.

10 Enfin, lors des recours (fréquents) en tribunal administratif contre l'avis du préfet, un resserrement sur les paysages dits « opposables » (juridiquement), essentiellement les paysages ou éléments de patrimoine inscrits ou classés, tend à s'opérer.

Figure 1. Procédure éolienne, degré de prise en compte du paysage dans l'instruction des projets éoliens et chronologie du projet Ventville

\begin{tabular}{|c|c|c|c|c|c|}
\hline Procédure éolienne & 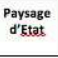 & $\begin{array}{l}\text { Paysages } \\
\text { partagés }\end{array}$ & $\begin{array}{l}\text { Ressources } \\
\text { paysageres } \\
\text { partagees }\end{array}$ & Acteurs Yeatville & $\begin{array}{l}\text { Chrono } \\
\text { Yeatsille }\end{array}$ \\
\hline $\begin{array}{l}\text { Exploration (potentiel de } \\
\text { vent, connexion au réseau, } \\
\text { contexte local ...) }\end{array}$ & + & & & $\begin{array}{l}\text { agriculteurs (dont maire), } \\
\text { développeurs }\end{array}$ & Fin 2003 \\
\hline $\begin{array}{l}\text { Installation d'un mát de } \\
\text { mesure }\end{array}$ & + & & & & - \\
\hline $\begin{array}{l}\text { Echanges avec } \\
\text { l'administration }\end{array}$ & +* & + & + & $\begin{array}{l}\text { administration, } \\
\text { développeur, } \\
\text { regroupement des projets }\end{array}$ & Fin 2003 \\
\hline Etuded dimpact & + & + & + & ballon sonde & Avril 2006 \\
\hline Réunion publique & + & + & + & développeur & Juin 2006 \\
\hline \multirow[t]{2}{*}{ Dépôt d'un projet de ZDE } & *+ & + & + & maire, développeur & $\begin{array}{l}\begin{array}{l}\text { Novembre } \\
2006\end{array} \\
\end{array}$ \\
\hline & & & & $\begin{array}{l}\text { réunion M. et Mme A, } \\
\text { maire, developpeur }\end{array}$ & Fin 2006 \\
\hline \multirow{8}{*}{$\begin{array}{l}\text { Dépôt d'une demande de } \\
\text { permis de construire }\end{array}$} & +* & + & + & & Janvier 2007 \\
\hline & & & & $\begin{array}{l}\text { réunions publiques APPGS } \\
\text { Gâtinais Sud, zones de } \\
\text { vigliance }\end{array}$ & Début 2007 \\
\hline & & & & $\begin{array}{l}\text { envoi de la pétition } \\
\text { (maire, préfecture, député } \\
\text { /CG) }\end{array}$ & Mars 2007 \\
\hline & & & & elections présidentielles & \begin{tabular}{|l|} 
Avril mai 2007 \\
\end{tabular} \\
\hline & & & & $\begin{array}{l}\text { validation de la ZDE par la } \\
\text { préfecture }\end{array}$ & 16 mai 2007 \\
\hline & & & & tensions, tags.... & Juin 2007 \\
\hline & & & & $\begin{array}{l}\text { Autorisation PC de la } \\
\text { partie du projet située } \\
\text { dans le Loiret }\end{array}$ & Février 2008 \\
\hline & & & & $\begin{array}{l}\text { élections municipales, } \\
\text { liste d'opposition }\end{array}$ & Mars 2008 \\
\hline Avis administratif $(P C)$ & + & + & + & & \\
\hline Avis CSPL & + & ++ & + & $\begin{array}{l}\text { comminssion CDNPS et avis } \\
\text { positif }\end{array}$ & \begin{tabular}{|l|l|} 
Juillet 2008 \\
\end{tabular} \\
\hline Enquête publique & +* & ${ }^{+}$ & + & $\begin{array}{l}\text { APPGS, associations et } \\
\text { collectifs locaux }\end{array}$ & Octobre 2008 \\
\hline $\begin{array}{l}\text { Avis du Commissaire } \\
\text { enquêteur }\end{array}$ & +* & +. & +. & avis positif & \begin{tabular}{|l} 
Automne \\
2007
\end{tabular} \\
\hline Pétition au préfet & + & + & + & avis positif & $-\quad$ \\
\hline Avis du préfet & + & + & + & préfecture & \begin{tabular}{|l|} 
Février 2009 \\
\end{tabular} \\
\hline Recours & & & & APPGS & $\begin{array}{l}\begin{array}{l}\text { Février } 2009 \text { - } \\
\text { mai } 2013\end{array} \\
\end{array}$ \\
\hline $\begin{array}{l}\text { Cour administrative } \\
\text { d'appel de Paris }\end{array}$ & & & & $\begin{array}{l}\text { autorisation définitive de } \\
7 \text { Éoliennes sur } 8\end{array}$ & Mai 2013 \\
\hline
\end{tabular}

11 Pour clarifier les abords du paysage dans ces processus, nous proposons tout d'abord la notion de « paysage d'État » (Nadaï, Labussière O. 2011, 2013). En France, l'approche de l'État en matière de paysage s'enracine dans une tradition administrative de gestion des monuments et de leurs abords qui remonte au début du $\mathrm{xx}^{\mathrm{e}}$ siècle et qui a jeté les bases d'une forme d'objectivation institutionnelle du paysage. La primauté du visuel et la géométrisation de l'espace (covisibilités, délimitation en plan par zonage) restent des moyens usuels de l'État pour traduire le paysage en «bien public» et légitimer son action. Le "paysage d'État", ainsi constitué d'une multitude d'aires protégées dont l'État est le garant, est une composante structurante du territoire. En ce qu'il recouvre des éléments paysagers ayant surtout une existence réglementaire, il ne se superpose que partiellement aux formes d'appropriation individuelle et collective du paysage. 
12 Cette tradition administrative de protection centralisée constitue le paysage d'État comme un commun au sens d'un patrimoine national dont la jouissance doit être assurée pour tous. D'autres formes d'appropriation du paysage, sans doute plus informelles même si elles peuvent être adossées à des formes élaborées de gestion, méritent d'être rassemblées et distinguées sous le terme de " communs paysagers ». Un premier degré d'appropriation correspond à des paysages faisant l'objet, comme tout paysage d'accès ouverts, d'un faisceau d'usages qui s'entrecroisent, se superposent sans que les uns ne limitent ou ne réduisent les autres. Comme nous le verrons dans le cas d'étude, la "plaine » est un territoire agricole vierge d'infrastructures et apprécié des agriculteurs au travail, des chasseurs comme des villageois pour leur promenade dominicale. Nos entretiens - ce n'était pas leur objet premier - ne nous permettent pas de restituer les formes éventuelles de coordination entre ces groupes d'usagers. C'est pourquoi nous nous en tenons à considérer le paysage comme une ressource partagée en commun au travers d'usages relativement informels. Un second degré d'appropriation correspond à l'association de la ressource paysagère à une forme d'organisation ou d'action collective qui en reconnaît et en régule les usages partagés en relation avec une activité spécifique. Cela se concrétise dans notre cas d'étude par le rôle d'une association foncière composée d'agriculteurs visant à organiser une répartition équitable des terres de la " plaine». Cette organisation collective autour de la question foncière peut être qualifiée, dans un sens proche de celui proposé par Elinor Ostrom (1990), de « bien commun » à dimension paysagère. Il ne s'agit pas d'une ressource en libre accès mais bien d'une ressource commune (partagée par les agriculteurs de la plaine) accompagnée d'une régulation auto-organisée, ses usages ayant un caractère rival et non exclusif.

\section{Politique éolienne, éolien politique}

13 La notion de commun paysager fait valoir dans l'analyse le paysage comme une ressource partagée au travers de modes d'appropriation directs (usages informels) ou indirects (exploitation agricole de la plaine). La question paysagère est plus difficile à positionner par rapport à la politique éolienne.

14 Cette dernière est une politique environnementale avant d'être une politique $d u$ paysage : elle se saisit de la promotion des énergies renouvelables comme moyen de lutter contre le réchauffement climatique. Le paysage pourrait être positionné comme une externalité de la politique éolienne. Cependant, une interrogation reste ouverte, relative au fait que les modes d'appropriation du vent ne sont pas caractérisés dans le droit (Le Baut-Ferrarese et Michallet, 2012). Le vent est une chose, ce n'est ni un bien ni un bien marchand: cette ressource reste, par son caractère insaisissable et sa profusion, en dehors du droit de propriété. Les doctrines du droit ne convergent pas sur le fait de présumer (ou non) un statut de chose commune, pour les choses non appropriables telles que le vent. Exclu de la cohorte des biens, le vent comme ressource reste communément appréhendé et défini par sa seule physique en tant que flux renouvelable. Ce vide juridique se double en France de la prédominance d'un éolien industriel de portage privé alors même que l'exploitation du vent suscite des impacts sur d'autres ressources communes.

15 La question paysagère naît de cette tension entre une ressource non qualifiée en droit (le vent) et son appropriation intensive à dimension territoriale. La privatisation du 
développement éolien en France s'accorde difficilement avec les organisations individuelles ou collectives qui règlent les partages des ressources territoriales sur lesquelles il a des impacts, dont les communs paysagers. Cette donne est compliquée par le fait que le paysage d'État n'est le plus souvent pas à même de réguler les enjeux associés aux paysages partagés qu'il ne recouvre pas. Plus encore, il est bien souvent pris en défaut dans son propre domaine de compétence (covisibilités avec des paysages " opposables »), du fait de l'étendue et de l'ampleur des covisibilités éoliennes (Nadaï et Labussière, 2011). Les enjeux de paysage éolien se heurtent donc à des formes de régulation inadéquates (une tradition française centralisée de protection des paysages) ou inexistantes (le vent comme ressource aux modes d'appropriation non régis par le droit). Il en résulte presque inévitablement une politisation des enjeux éoliens au niveau local au sens d'une recomposition de collectifs autour d'enjeux de paysages. Cette politisation emprunte des chemins multiples. Elle questionne les projets tout autant que les modes de régulation qui en fondent le développement, qu'il s'agisse des politiques publiques (politiques éolienne ou du paysage) ou encore de l'articulation entre sphère publique et sphère privée au niveau local.

\section{Suivre les communs paysagers : le projet éolien et son « public »}

La question paysagère permet d'analyser la politisation des enjeux éoliens à partir d'un entre-deux analytique, au croisement de problématiques privée et publique. Il s'agit de suivre la destinée des communs paysagers à la rencontre d'un éolien de portage privé, qui exacerbe le manque de cadrage juridique des ressources qu'il requiert (le vent et, par extension, le foncier, etc.), et d'institutions (dont le " paysage d'État ») en charge de réguler ou de protéger ces ressources.

Il faut reconnaître à Elinor Ostrom (1990) le dépassement de la dichotomie régulation privée (par allocation de droits de propriété)/régulation publique (par une agence centrale externe) au profit d'une analyse de formes auto-organisées de régulation des ressources communes. Dans notre cas, les formes d'appropriation étant en partie informelles, une analyse par la rationalisation des choix collectifs selon l'appareillage théorique d'Ostrom paraît difficilement praticable (Coriat, 2013; Ostrom et Basurto, 2013). Cette configuration empirique appelle donc une autre manière de comprendre l'émergence ou non de partage collectif et le devenir des communs paysagers existants.

L'analyse qui suit tente cet exercice en s'inspirant de notions d'«enjeu » et de "public», empruntées au pragmatisme, et proposées par Noortje Marres (2007). Comme le montre cette auteure, un point de départ intéressant dans l'analyse des processus de politisation est la notion d'enjeu : issues ou matters of concern, en anglais. L' issue désigne l'état indistinct d'un enjeu lorsque celui-ci ne peut être clairement pris en charge par des pratiques, des catégories ou des codes en place (économiques, politiques, scientifiques). Comme son nom l'indique en anglais, son destin est de trouver une voie de sortie, un mode de prise en charge ${ }^{3}$.

Le moment d'émergence de l'enjeu correspond à un moment politique : celui de la constitution de ce que Dewey appelait un "public» au sens d'un problème, d'un collectif concerné par cet enjeu et qui cherche à le porter en partage, à le rendre public. Noortje Marres insiste sur le fait que la constitution d'un enjeu en problème requiert un travail collectif de construction. Ce registre d'émergence, que Bruno Latour (2007) désigne comme le « politique 2 », est celui dans lequel l'intérêt général associé à l'enjeu 
n'est pas stabilisé : il est en question. Tout autres sont les registres du politique dans lesquels l'intérêt en jeu étant stabilisé, se pose plutôt la question de l'institution ou de l'organisation à même de le prendre en charge. Faute d'un fondement clair, les interactions entre les communs paysagers, un éolien de portage privé et le paysage d'État relèvent du « politique 2 ».

Le cas d'étude abordé ici porte sur l'émergence d'un projet éolien et des réseaux d'acteurs qui, de 2006 à 2009, en viennent à le contester et à s'opposer au développement éolien à l'échelle du plateau du Gâtinais sud (Seine-et-Marne). La pratique politique locale repose par tradition sur une subtile articulation entre sphère privée et vie publique villageoise : l'électeur est un voisin, l'agriculteur est un collègue et un promeneur du dimanche, l'opposant à l'éolien est un ami. L'enjeu que fait peser l'éolien sur les communs paysagers appelle une forme d'action collective qui redispose inévitablement les articulations en place. L'intérêt du déplacement vers les notions de public et d'enjeu est de pouvoir suivre le devenir de l'enjeu de paysage soulevé par le projet éolien, dans ses tentatives de se constituer comme problème partagé. Ce cadre analytique permet aussi de suivre cet enjeu sans le détacher de la vie politique locale qui distribue les sphères publiques et privées pour s'y articuler, ni de la politique éolienne et du paysage d'État qui proposent une définition et une pratique de l'intérêt général. Ce faisant, l'analyse s'inscrit dans le prolongement des travaux abordant le paysage comme projet et assemblage sociaux (Briffaud, 1995 ; Trom, 1996; Dewarrat et al., 2003 ; Nadaï, 2007b ; Nadaï et Labussière, 2010, 2013 ; Labussière et Nadaï, 2014)

21 Notre analyse repose sur une base de trente-quatre entretiens semi-directifs avec des services de l'État, des associations d'opposants ou des particuliers opposés au projet ainsi que des élus locaux opposés ou favorables à l'éolien. Ce travail comprend également des entretiens in situ, avec des particuliers, pour saisir les formes d'attachement à leur espace de vie ou de travail. Enfin, nous avons invité les interviewés à tracer sur des cartes leur aire de vigilance dans l'opposition à l'éolien puis à la discuter en fonction de leurs pratiques spatiales ou des paysages.

\section{Le difficile devenir public d'un projet éolien}

\section{Tension sur le paysage d'État, un projet sans public}

Fin 2003, deux agriculteurs de Ventville, une petite commune du sud de la Seine-etMarne, contactent une société spécialisée dans le développement de projets éoliens industriels. Pour l'administration de Seine-et-Marne, ce projet éolien est «une première ${ }^{4} »$. Soucieuse d'en garantir la qualité, elle demande aux développeurs présents sur le secteur de se grouper pour porter un projet sur trois communes: Ventville, Soville et Gatenville ${ }^{5}$.

Comme ailleurs en France, l'éolien met en difficulté la gouvernance du paysage. Le Service départemental de l'architecture et du paysage (SDAP) de Seine-et-Marne en témoigne :

« Des machines de 150 mètres, c'est hors d'échelle par rapport à tout ce qu'il y a sur

le département ${ }^{6}$."

Pour pallier cette difficulté, l'administration inaugure un nouveau procédé. En avril 2006, un ballon-sonde de couleur blanche est hissé à la hauteur des mâts des futures éoliennes. Il matérialise in situ la présence des éoliennes. Durant une journée, la 
direction régionale de l'Environnement (DIREN), le SDAP et la direction départementale de l'Équipement (DDE) sillonnent en voiture le territoire concerné par le projet. Il s'agit de traquer les covisibilités du ballon-sonde avec le patrimoine naturel et culturel, ainsi qu'avec les habitations. Cette expérience, qui permet de négocier des ajustements au projet et redonne à l'administration une capacité de maîtrise là où sa gouvernance du paysage était mise en échec, n'est pas portée à la connaissance des riverains.

Figure 2. Ballon-sonde blanc hissé au voisinage de Ventville

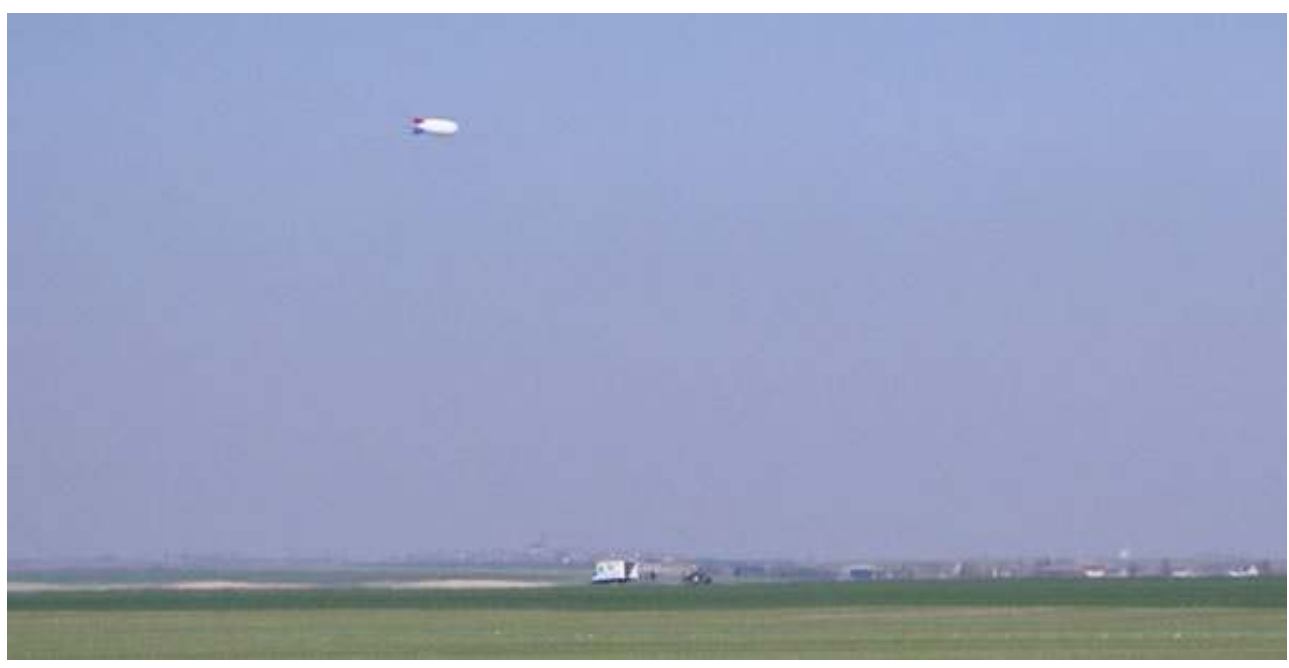

Comme l'illustre l'expérience du ballon-sonde, l'éolien opère, du fait de son dimensionnement, une tension sur les pratiques du paysage d'État. Il soulève un enjeu cognitif : comment appréhender en situation (visualiser) la présence des futures éoliennes (Nadaï et Labussière, 2011) ? Il met aussi en tension les principes et les hiérarchies de valeur, historiquement constitués, qui sont au cœur de ce paysage. Le paysage d'État pouvait jusqu'alors faire opérer ses hiérarchies de manière positive, sur le mode de la protection sélective d'éléments de paysages les plus valorisés : on arrêtait certains projets. L'éolien étant une infrastructure spatialement et visuellement colonisatrice, il ne s'arrête pas à cette protection et va chercher, sur des territoires entiers, les paysages moins valorisés pour s'y déployer. Il convoque ce faisant ces hiérarchies dans leurs effets induits pour les traduire en développements de fait: qualifier certains paysages, c'est en disqualifier d'autres dès lors que cette qualification oriente les développements de projets vers d'autres communs ou ressources paysagères partagées. Le paysage d'État peine à s'ouvrir pour tenter de se recomposer autour de ces nouveaux enjeux. Par rigidité, mais aussi pour ne pas être aux prises avec une politisation des valeurs qu'il est censé défendre en toute objectivité, il maintient, même quand il innove (e.g. ballon-sonde), une distance vis-à-vis du public.

Localement, le projet éolien a un statut ambigu. Les initiateurs du projet sont deux agriculteurs disposant du foncier suffisant pour implanter les éoliennes. Ils se sont adressés directement à une entreprise privée, sans solliciter l'appui d'une collectivité locale. Un des porteurs du projet n'étant autre que le maire de Ventville, cette initiative ne peut manquer d'interférer avec la gestion politique locale. En tant que maire, il est tenu d'informer les habitants de sa commune de tout projet industriel en voie de réalisation ou encore les communes voisines de tout projet éolien pouvant générer des covisibilités dans le paysage. Dans les premières années du projet, cette ambiguité n'est pas tranchée. 


\section{Un privé « en demande » qui ne se constitue pas d'emblée comme public}

Du côté des habitants de Ventville, les premières personnes à apprendre l'existence du projet sont M. et Mme A. Leur maison serait la plus proche des futures éoliennes. Ils sont donc concernés à titre privé par ce projet. À cette époque, ils n'ont pas de position arrêtée sur l'éolien. Durant une journée, ils se rendent sur la commune de Janville en Eure-et-Loir et pique-niquent au pied d'éoliennes :

«On a voulu voir la situation de vie dans cet endroit-là... c'était surnaturel! On

avait l'impression d'être dans un autre monde ${ }^{7}$."

De retour chez eux, ils s'informent par internet sur l'éolien et ses effets, nouent quelques contacts avec des associations d'opposants (Aude, Lévézou, Vent de Colère). Ils vont s'informer en mairie. Le maire accède à leur demande. Le projet devient plus concret.

M. et Mme A. entrent progressivement dans une posture de vigilance. Ils assistent à tous les conseils municipaux, premier lieu où $\mathrm{M}$. et Mme A. tentent de donner à leur expérience privée une dimension publique: il s'agit de rendre le projet éolien discutable et négociable.

En juin 2006, une réunion publique d'information est organisée par le développeur dans le hameau le plus proche des éoliennes. Fin 2006, le maire organise une réunion informelle entre le développeur éolien et M. et Mme A. Il informe ces derniers qu'une proposition de zone de développement éolien (ZDE) a été déposée par le conseil municipal à la préfecture le 20 novembre 2006 :

«Là on a compris que le projet était assez avancés. "

Bien que rarement concertées, les $\mathrm{ZDE}^{9}$ sont néanmoins une occasion inédite, dans l'état actuel des procédures administratives, pour traduire la politique éolienne française en orientations partagées au niveau local (Nadaï et Labussière, 2009). M. et Mme A. demandent au maire d'organiser un débat public sur l'opportunité du projet.

« La machine était enclenchée, il ne voulait pas ${ }^{10}$.»

En janvier 2007, le permis de construire vient d'être déposé par le développeur éolien à la préfecture. C'est une étape importante dans la formalisation du projet. Là encore, les communes n'ayant pas la compétence pour autoriser ou refuser le permis de construire éolien, cette décision ne peut contribuer à la mise en débat au niveau local.

M. et Mme A. organisent à leur domicile une consultation. Ils rassemblent des documents sur l'éolien et proposent à qui veut venir de signer une pétition. Si le paysage n'est pas mentionné dans les termes, le «risque de défigurer notre environnement» renvoie bien à l'idée d'un paysage local, que la pétition ouvre à un statut collectif. Bien qu'initié dans l'intimité du chez-soi, ce dispositif a une portée politique: il s'agit de constituer un collectif capable de soutenir une demande de référendum sur l'opportunité du projet. La pétition compte 70 signataires sur les 354 habitants de Ventville, soit $1 / 5$ de la population communale.

«Au départ [l'éolien] on en parlait. En signant la pétition, c'est devenu un engagement ${ }^{11} »$ raconte une signataire.

31 À la mi-mars 2007, la pétition est envoyée au maire de Ventville, à la préfecture de Seine-et-Marne, au député et au conseiller général. Cette nouvelle tentative pour donner à la question éolienne une dimension publique et négociable au niveau local 
reste sans suite, mais le maire est stupéfait. La liste des pétitionnaires comporte " certaines personnes qu'[il] connaissai[t] très bien ${ }^{12}$ ». En n'associant le maire qu'une fois l'agencement constitué et au titre de ses responsabilités publiques, ce collectif éolien déplace l'ordinaire de la régulation sociale à Ventville.

\section{L'articulation privé/public, un commun relationnel en question}

«Localement, c'est pas politisé du tout. C'est familial [...] même dans les villages environnants, c'est comme ça. C'est une "politique agricole ${ }^{13}$."

L'expression de "politique agricole » exprime la prédominance de familles anciennes du village, à la tête de grandes exploitations agricoles, sur la gestion politique communale. Les fonctions de maire et de conseiller municipal tiennent quasiment du legs générationnel.

Au-delà du conseil municipal, ces schémas familiaux et socioprofessionnels sont également structurants de la vie associative de Ventville.

«M. C., c'est le maire de Ventville, son père, c'est le président de l'association de chasse, sa mère, c'est la présidente du club des anciens, sa femme, c'est la présidente du comité des fêtes. C'est comme ça dans les petits villages. C'est leur monde à eux [les agriculteurs]. »

Cette collusion du milieu agricole avec les responsabilités locales fait que depuis plusieurs décennies la vie municipale puise sa vitalité dans des cercles familiaux cohérents et influents.

Tout en favorisant une reproduction des élites locales, cette structure fut longtemps vertueuse. Elle se présentait comme un jeu ouvert assurant la bonne réputation du village et son animation: les nouveaux venus étaient accueillis et aidés, ils s'engageaient à leur tour dans l'organisation de la vie locale, schéma auquel ont adhéré M. et Mme A. Ainsi, un certain nombre de réciprocités étaient-elles rendues possibles entre une gestion politique relativement circonscrite et une vie sociale plus ouverte favorisant l'investissement des individus.

L'éolien intervient donc dans un tissu social où la relation politique s'opère d'ordinaire dans la proximité, le maire tenant le rôle de chef d'orchestre. Il déstabilise ce jeu coopératif qui, dans son rôle de régulation, constitue un véritable commun relationnel.

La pétition constitue un nouvel espace politique. La demande de référendum et la dimension publique, jusqu'alors révélées à l'occasion de réunions d'intérêts privés (réunion informelle de M. et Mme A. avec le développeur et le maire ; consultation chez M. et Mme A.), acquièrent une présence nouvelle. La dimension publique du projet n'est plus uniquement procédurale (gouvernance paysagère de l'État, élaboration de la ZDE par le conseil municipal), elle acquiert une épaisseur politique (portage par le maire).

La pétition renvoie le maire à son rôle d'élu local, ce dont il tente de tirer parti. Quand « on est élu, on a des projets. S'ils ne vous plaisent pas, il y a les élections pour nous mettre dehors. C'est ça la démocratie locale pour moi $^{14} »$. Le maire fait comme si le projet éolien correspondait à un intérêt public constitué alors que ce projet n'a jamais été au cœur de son programme électoral. Il est ce faisant pris au piège. En s'arc-boutant sur son mandat pour affirmer sa légitimité à décider du projet éolien, le maire se coupe de sa relation politique ordinaire : cette proximité qui fait sa légitimité et lui donne accès aux enjeux au travers de qualités qui les rendent négociables.

S'agissant de M. et Mme A., le projet éolien est négociable : 
« Nous, on ne s'est jamais engagé contre les éoliennes mais contre ce projet ${ }^{15}$. » Leur accord est conditionné à la modification de quelques implantations d'éoliennes, «la seconde, par exemple, était trop près [de chez eux $]^{16}$ ». Si le référendum avait eu lieu et avait débouché sur un plébiscite, ils auraient accepté l'implantation des éoliennes. « Nous, on aurait mis la maison en vente, mais ça devenait notre problème à nous ${ }^{17}$ ", mais là, " on n'a pas été associé du tout ${ }^{18}$ ».

La défense du maire ne parvient pas à dissiper l'ambiguïté qui le lie depuis le début au projet éolien. Les modalités de mise en partage de ce projet - et des paysages qu'il emporte - restent à définir.

\section{La plaine, tension sur un commun agricole}

La «plaine » de Ventville est un cas d'espèce. Espace agricole de plusieurs centaines d'hectares, elle a été récemment remembrée sans conflits. Comme l'explique M. L., président de l'association foncière et de remembrement de Ventville, sa qualité tient à ses remembrements successifs, qui ont permis de rendre aux exploitations une unité s'effaçant au fil des successions et des cessations d'activité. La configuration même de la plaine (topographie régulière, grande taille, qualité homogène) facilite cet exercice de mise en équivalence/ré-allocation des parcelles, souvent source de tensions entre agriculteurs.

«C'est précisément pour tout ce fonctionnement que l'éolien devient problématique."

Pour le prochain remembrement, M. L. soulève de nombreux points d'interrogation : destination des loyers éoliens lors de la cessation de parcelles, câblages enfouis, devenir des infrastructures en fin de vie ou si le développeur actuel fait faillite...

En d'autres termes, le contrat éolien introduit un rapport à la terre qui fait fi de la responsabilité collective des agriculteurs à l'égard de la plaine et qui ne semble pas vraiment suivre les usages de la transmission patrimoniale. Les contrats sont signés individuellement et dans le secret avec chaque agriculteur, l'association foncière n'est pas consultée, les agriculteurs contractants engagent toute leur exploitation sans savoir précisément où seront localisées les éoliennes. L'éolien n'introduit pas seulement une forme de privatisation de l'espace, là où prévalait une gestion communautaire, il provoque localement une situation d'ingérence.

«D'habitude le maire ne décide rien sur ce qui se passe dans la plaine, il est plus

logique que ce soit le président de l'association."

En soutenant ce projet éolien privé adossé à quelques hectares de plaine, la mairie intervient comme par effraction dans une communauté agricole souveraine.

\section{Se constituer comme public, le plateau du Gâtinais sud comme commun et espace politique éolien}

La pétition portée par le collectif de Ventville a peu d'effets. Néanmoins, elle lui permet de prendre contact avec différentes associations d'opposants du sud seine-et-marnais (l'APPGS, l'ADER) et du nord du département (Vent de Vérité). L'Association de protection du plateau du Gâtinais sud (APPGS), créée à l'origine par des habitants d'une commune voisine, en janvier 2007, devient rapidement l'instance de coordination de ces différents collectifs. 

politique éolienne française :

«L'éolien industriel est une manne financière pour des gens peu scrupuleux ${ }^{19}$." Le président de l'APPGS insiste sur ce point :

" Je vais même excuser les maires. L'État a laissé faire. Dans les petites communes, les maires n'ont pas grand-chose à gérer, un projet éolien, ça les dépasse ${ }^{20}$. 》

Ce faisant, l'APPGS opère d'emblée dans le champ politique en mettant les maires en demeure de prendre position sur l'éolien.

Au début de l'année 2007, l'association organise deux à trois réunions publiques par semaine. Tout le Sud de la Seine-et-Marne est couvert. L'APPGS obtient de nombreuses communes sollicitées par des développeurs qu'elles prennent position contre l'éolien. Ce faisant, elle fait du développement éolien le problème d'un territoire beaucoup plus vaste : le Sud du département.

La production de cette échelle de vigilance constitue un processus novateur. Elle témoigne de la recherche d'un espace de référence au sein duquel les mutations territoriales et paysagères de l'éolien seraient collectivement négociables. Le plateau du Gâtinais sud constitue une spatialité politique nouvelle. Non que l'APPGS n'existât pas avant l'enjeu éolien. Pour autant, l'émergence d'une veille stratégique partagée de la part des membres de l'association, associée à une conscience spatialisée des zones de responsabilité, bien traduite par les espaces et pratiques de vigilance adoptés par les habitants de ce secteur, peut être considérée comme une forme d'organisation collective.

Figure 3. Zone de vigilance contre l'éolien de Mme A. (tracé)

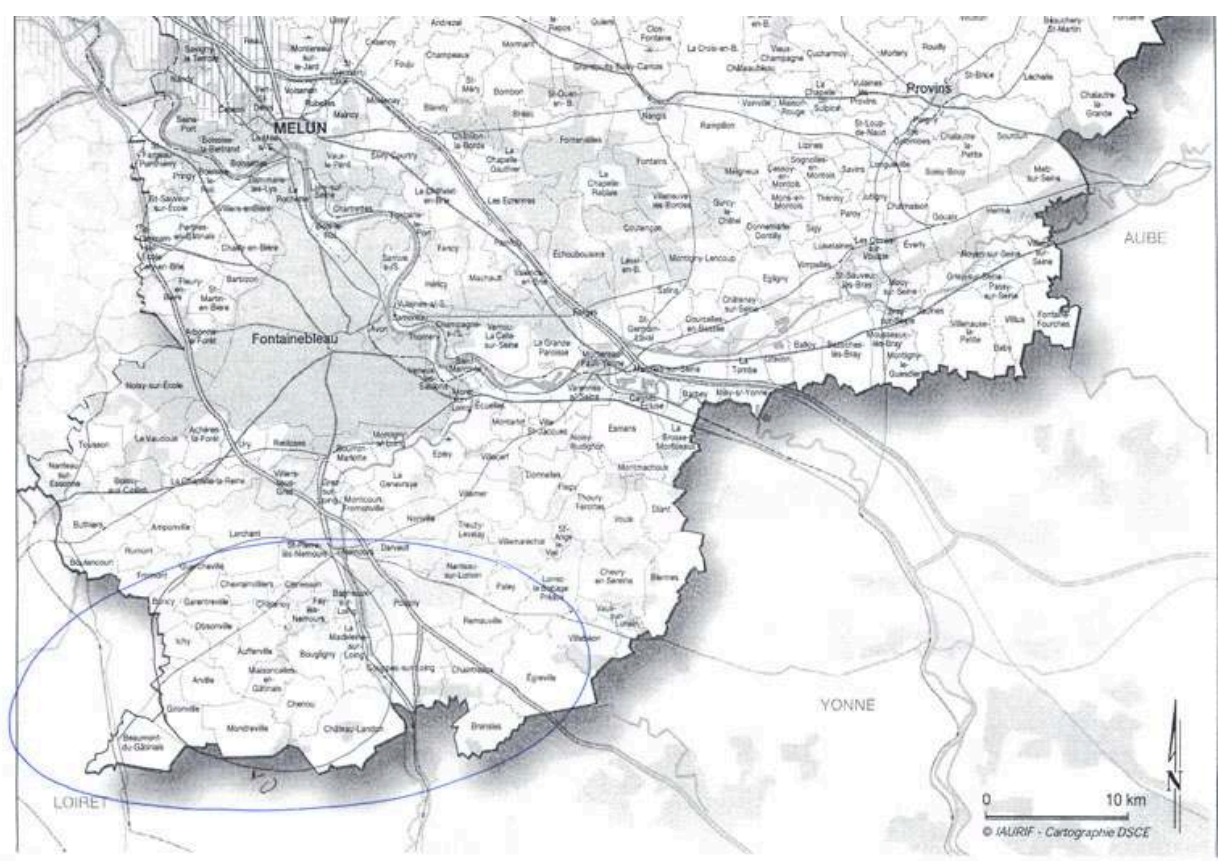

«Tout ça c'est ma zone [de refus de l'éolien]. Donc là c'est régional. J'ai déjà arpenté tout ça ${ }^{21}$. » 
Figure 4. Zone de vigilance contre l'éolien de Mme N.

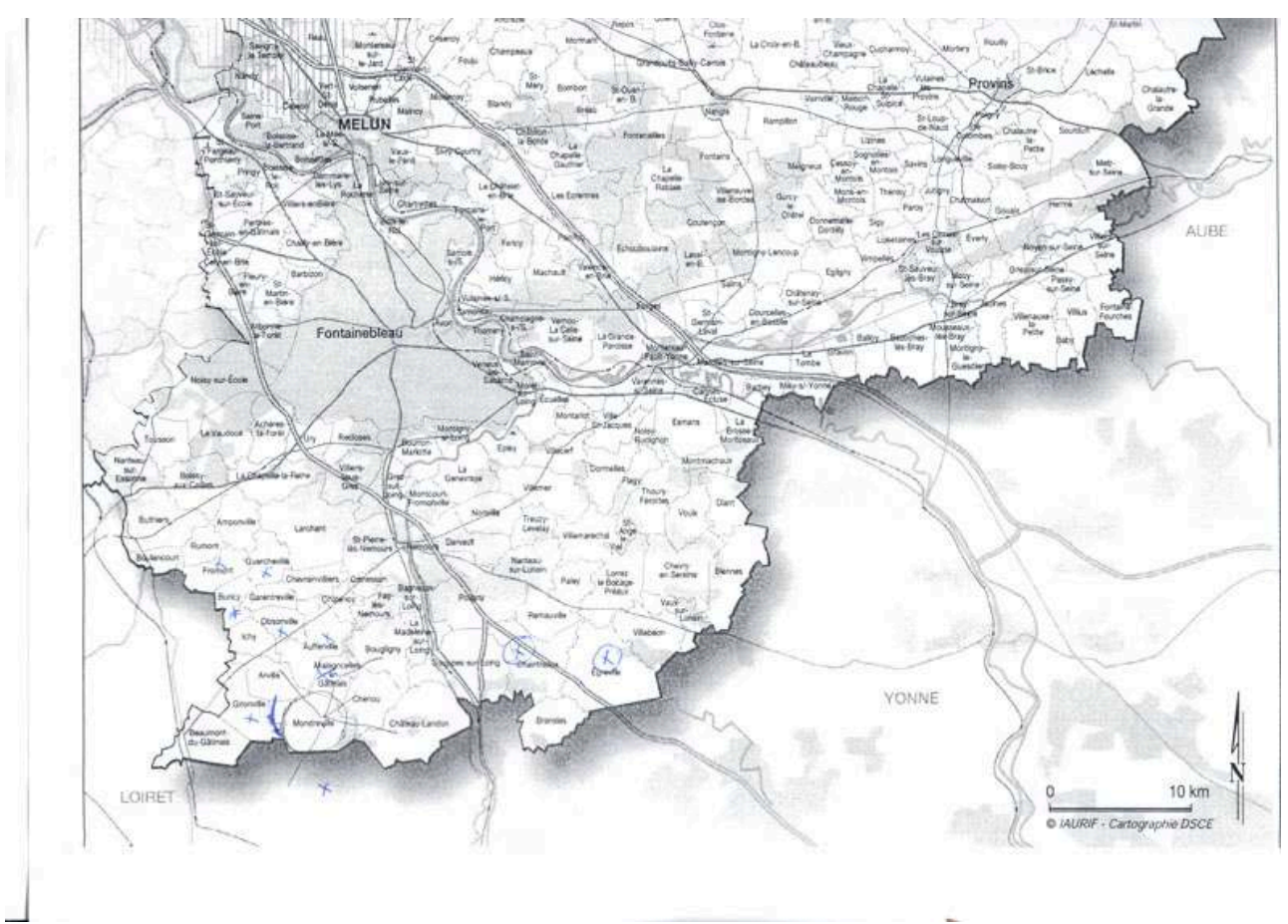

" Dès que l'éolien était à l'ordre du jour d'un conseil municipal, on y allait. On patrouillait. Là vraiment j'étais opposante. On se disait qu'il fallait faire capoter les projets autour pour que le nôtre ait une chance de ne pas voir le jour ${ }^{22}$.»

Elle consacre le passage de ce plateau du statut de ressource partagée à celui de commun. Un commun certes émergent, et peut-être temporaire, mais un commun porteur d'une remise au travail de la politique éolienne française comme politique d'offre sectorielle n'offrant aucune possibilité d'arbitrage local sur le bouquet énergétique.

Pour le maire de Ventville, cette période est «très mal vécue ${ }^{23}$ ». Alors que les réunions d'opposants à l'éolien se multiplient, il souhaite organiser une réunion publique avec l'appui des agents de l'État, mais à l'approche des élections (présidentielles en avril et mai 2007, législatives en juin), l'administration reste en retrait de dossiers politiques tels que l'éolien.

\section{Advenir comme public, radicalisation et dislocation de la vie villageoise}

Si l'APPGS démultiplie l'opposition à l'éolien avec une certaine efficacité, elle laisse entière la demande de référendum portée par le collectif d'habitants de Ventville. La radicalisation, facilement mobilisable pour advenir comme public, est aussi un piège : une fois constitués comme groupe d'opposition, il est plus difficile aux opposants de faire valoir leur souhait premier de négociation.

Les témoignages recueillis montrent en effet des individus qui ne sont pas opposants en soi à l'éolien mais qui se sont construits une posture d'opposition, souvent à la suite d'un manque de réponses de la part des dispositifs institutionnels et politiques. 

de la vie sociale. Le constat est amer :

« Notre village était réputé pour avoir une très bonne ambiance ${ }^{24}$."

Le village a littéralement implosé. Nos entretiens montrent qu'il y a un avant et un après l'éolien.

En l'absence de référendum, M. et Mme A., de même que plusieurs habitants, manifestent publiquement leur opposition.

«On a mis des palettes avec "Non aux éoliennes", des trucs comme ça. Mais on a demandé aux propriétaires pour les mettre, on a averti le maire, on a fait les choses proprement $^{25}$. »

Figure 5. Panneau de protestation contre le projet éolien à l'entrée de Ventville

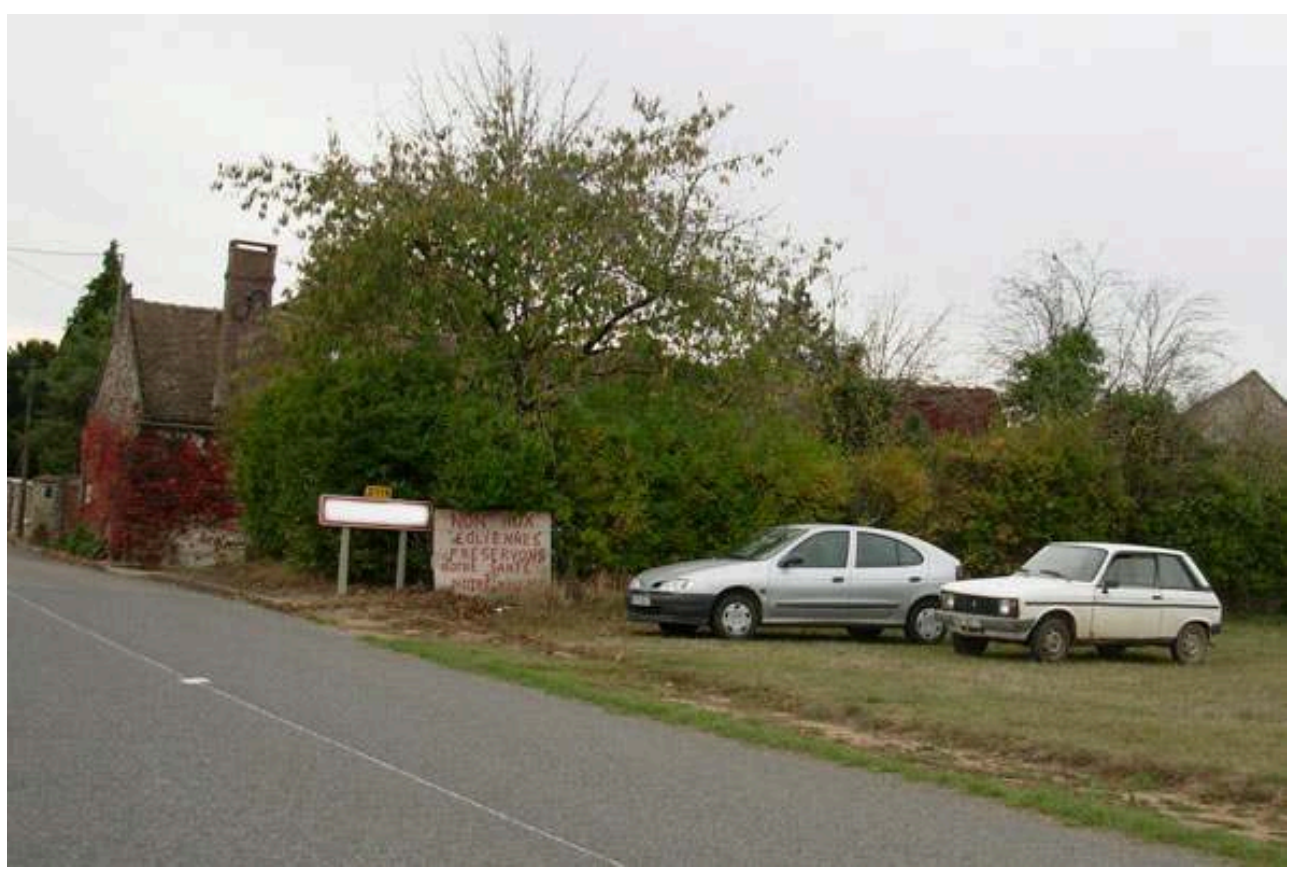

L'animosité qui entoure le projet en 2007, après l'échec de la pétition et la validation de la ZDE par la préfecture (16 mai), donne lieu à des débordements. Un matin de juin 2007, les habitants découvrent des tags au cœur du bourg. M. et Mme A. sont convoqués à la gendarmerie. Ces derniers, ainsi que le maire, subissent des jets de bouteilles et de poubelles sur leurs habitations. Les fauteurs de trouble ne sont pas identifiés.

Cette confusion débouche progressivement sur une dislocation de la vie sociale et un désengagement des activités collectives :

«Au niveau de Ventville, on n'a plus de vie publique, tout ça s'est cassé26. »

"Avant il y avait des politiques droite - gauche, comme partout, maintenant l'éolien a tout mélangé, ça a créé des fractures entre copains, ça a aussi rapproché des gens qui ne pouvaient pas se voir ${ }^{27}$." 


\section{Des procédures improductives pour doter l'éolien d'une dimension publique} quelques avis d'opposants avec motivations et seulement une dizaine de pour, dont des conseillers municipaux de Soville ${ }^{31}$ ", le commissaire enquêteur émet un avis favorable. La désillusion est totale :

« Il [le commissaire enquêteur] a fait un amalgame en disant que vu le résultat des élections municipales, le maire largement réélu, donc de toute façon, les antiéoliens... une enquête publique avec un rapport comme ça, non, ça n'est pas une enquête publique ${ }^{32}$."

Les mêmes opposants reconnaissent que lors de l'enquête publique d'une commune voisine, le commissaire enquêteur avait fait un travail satisfaisant, bien qu'il eût rendu un avis favorable. Le 11 février 2009, la préfecture accorde le permis de construire pour les huit éoliennes de Ventville et Soville. L'APPGS engage plusieurs procédures visant à annuler cette décision, sans succès. En mai 2013, 7 des 8 éoliennes sont définitivement autorisées au permis de construire. L'éolienne exclue l'est en raison d'un survol non maîtrisé. La livraison du parc est prévue à l'automne 2014 pour une mise en service fin 2014.

59 Au final, les différents dispositifs constitués par les élections municipales, l'enquête publique, le recours au tribunal administratif ne parviennent pas à conférer une dimension négociée et partagée au parc éolien. Bien au contraire, les élections semblent n'offrir que des thérapeutiques faussées, avant tout destinées à traiter un social «problématique» et laissant impensée la technologie éolienne dans les 
recompositions qu'elle suscite, qui pourtant sont à l'origine de nombreuses désorganisations locales.

\section{Un éolien hors du commun}

60 L'analyse qui précède montre comment un projet éolien suscite la déstructuration de plusieurs types de communs: un commun relationnel fait de l'intime entrelacement des intérêts publics et privés au sein du village de Ventville, des communs paysagers résultant de la cohabitation des usages informels (promenades) ou institutionnalisés (agriculture, remembrement) de la « plaine ».

61 À la lumière de ce constat, on comprend qu'un certain type d'éolien (portage privé, capitalistique), sans épuiser le vent et tout en pouvant se prévaloir d'un caractère renouvelable, va épuiser des communs qui sont des ressources locales structurantes de la vie villageoise. Le vide juridique qui tend à faire du vent une chose qui n'appartient à personne et dont l'usage est commun à tous n'interrompt pas dans les faits la concurrence qui s'instaure entre l'éolien et d'autres usages pour des ressources qui conditionnent l'exploitation du vent. Le paysage paraît ainsi bien étranger à l'exploitation du vent comme flux physique renouvelable et pourtant il fait partie des choses sur lesquelles un développeur éolien prend appui au travers d'un faisceau d'usages associés au foncier, à l'organisation de l'espace, à la productivité des terres, etc.

62 Pour comprendre la nature des concurrences qui s'instaurent autour de ces ressources, il faut rappeler deux éléments relatifs aux politiques de l'éolien en Europe et en France. En Europe, la constitution de l'éolien comme politique publique a débuté par un âpre débat sur les formes de portage et les instruments économiques de soutien à cette technologie (i.e. tarif, prime, quota, certificat). La politique éolienne a en quelque sorte émergé au milieu du gué : elle a laissé en amont, indiscutée, la question du statut de la ressource, de ses modes d'appropriation, rendant souvent problématique en aval la question de l'équitable répartition des bénéfices retirés de l'exploitation du vent. En France, cette donne est rendue plus compliquée par la prédominance du portage privé au travers duquel l'éolien noue et dénoue les ressources et les éventuelles organisations collectives qui les sous-tendent. Cet éolien-là est supposé pouvoir se développer librement, sur le mode de l'intéressement privé, sans avoir à opérer un coûteux travail de gestion collective de la ressource.

63 Face à cela, la capacité des institutions à faire émerger un intérêt partagé autour des projets éoliens est faible. La politique éolienne française possède une dimension publique (objectifs quantitatifs, tarif d'achat) mais elle relègue au niveau local, sans vraiment l'accompagner, un travail de coordination collective seul à même de constituer un intérêt public autour de l'éolien. Ainsi, le décret qui fonde la ZDE évoque les communs paysagers (paysages classés, inscrits ou partagés) sans soutenir les collectivités ou l'administration dans la définition du partage qui est en jeu. En Seineet-Marne, l'administration instruit la ZDE puis le projet éolien de façon technique : elle renvoie les tensions que ces dispositifs suscitent à une prise en charge plus tardive (enquête publique, pétition au préfet, recours auprès du tribunal administratif). Ni l'opérateur privé ni l'administration ne parviennent à revenir sur l'amont de la politique éolienne de manière à ouvrir un espace de discussion sur les ressources en jeu, les organisations qui les instituent comme communs partagés et à réfléchir à 
nouveau frais aux formes d'appropriation et de répartition à mettre en place. Les élus se retrouvent seuls face à leurs électeurs pour assumer les tensions suscitées par un éolien constitué de fait comme objet de portage privé.

S'agissant des biens communs, Elinor Ostrom (1990) souligne la distinction fallacieuse entre modes de régulation publique et privée. Notre analyse rejoint ce constat en montrant que la politique éolienne française possède une double dimension publique/ privée (objectifs quantitatifs et tarifs d'achat; appel aux développeurs privés pour réaliser cette politique). Cet entrelacement ne rencontre pourtant aucune prise en charge. Les dimensions publique et privée de la politique éolienne font l'objet de tentatives de prises en charge disjointes : les développeurs privés ciblent des ressources en les déliant des collectifs qui les pérennisent; les administrations, par souci de neutralité, mettent en œuvre des procédures sans se mêler des tensions entre intérêts privés qu'elles suscitent. Sur le plan du paysage, il en résulte des prises en charge inadéquates (protéger le "paysage d'État» sans tenir compte des conséquences de cette protection) ou inexistantes (faire du vent une ressource isolée, réduite à sa physique). En l'état, les communs (relationnel villageois, paysager) en ce qu'ils reposent sur un entrelacs d'intérêts publics et privés ont peu de chance d'être pérennisés. Ainsi, le type d'éolien auquel nous sommes confrontés dans ce cas d'étude partage un grand nombre de collectifs sur son passage en rendant les intérêts publics ou privés incommensurables les uns aux autres. Il déclenche des processus que nous pourrions qualifier de fin des communs. La vie sociale à Ventville se délite parce que les familles agricoles qui l'animaient et tiraient leur influence de la superposition entre logiques familiales (privé) et responsabilités municipales (public), sont contrecarrées par l'éolien, en tant qu'il fait tomber l'intéressement privé dans le champ du profit capitaliste et met les élus face à des responsabilités outrepassant la gestion ordinaire. De même, s'agissant de la gestion de la plaine et de l'organisation de la vie agricole, l'éolien met en échec le fonctionnement d'un commun. Les développeurs introduisent une logique d'appropriation de la terre (implantation pérenne, location, transmission incertaine) qui soustrait l'agriculteur contractant à la gestion communautaire du foncier agricole (qualité globale de la plaine, échanges de parcelles et remembrements) et produit des externalités négatives (câblages, « zone morte » de l'éolien).

Nous évoquions la difficulté à " remonter » vers l'amont au sens d'une discussion qui rouvre le statut accordé à la ressource pour juguler cette fin des communs. Ce n'est pas la seule piste. Il s'agit aussi de reconnaître, comme nous l'enseigne l'observation du fonctionnement de communs paysagers, les densités d'usages dont ils sont porteurs. Ces usages se caractérisent par des attachements qui les lient à des entités variées (productivité agronomique des sols, qualité paysagère, biodiversité, etc.) composant une certaine configuration paysagère. Ceci suggère d'aborder la ressource en vent, au premier abord si fluide et donc si peu territoriale, à travers l'analyse de configurations paysagères spécifiques comprises comme des faisceaux d'usages structurés par des collectifs et des porte-parole vis-à-vis desquels l'éolien peut entrer en rivalité. L'exercice est délicat à stabiliser car l'éolien tend à bousculer les échelles d'appréhension du paysage et les façons de l'apprécier collectivement ${ }^{33}$. Dans notre cas d'étude, le devenir du plateau du Gâtinais sud illustre l'émergence d'un nouveau commun paysager porteur d'une recherche de régulation collective alors que celle-ci est absente à un niveau mésoterritorial. 


\section{Conclusion} se construisent une posture d'opposition à mesure que les dispositifs politiques et institutionnels qu'ils sollicitent font défaut. Ce ne sont pas des opposants de principe et ils n'ont pas de position définie par rapport à l'éolien lorsque le projet émerge. Les oppositions se construisent à partir des tensions qu'exerce l'éolien (français) de portage privé sur les communs (relationnel villageois, paysager). Si ces tensions induisent une fragmentation de certains communs à travers un dérèglement de l'articulation entre sphères privée et publique qui sous-tendait le lien social, elles suscitent aussi de nouvelles articulations et inaugurent l'émergence de nouveaux communs, en vue d'interroger la politique éolienne française dans sa capacité à se constituer comme dimension partagée.

Comprendre l'ampleur des tensions sociales que provoque l'éolien en France passe par une compréhension de la difficulté de la politique éolienne à articuler ses versants public et privé. Le cas d'étude présenté suggère que les oppositions locales seraient moins des intérêts privés opposés à une politique publique, comme pourrait le suggérer leur assimilation si souvent invoquée à du Nimby ${ }^{34}$, que le symptôme d'un environnement institutionnel inadéquat à accompagner les recompositions sociales que l'éolien suscite.

Cette dimension n'en reste pas moins à construire à mesure que l'éolien se territorialise. L'articulation des versants public et privé de cette politique éolienne se joue aussi (si ce n'est surtout) au niveau local, où les communes se retrouvent en position de piloter par elle-même, sans réelle médiation publique, les processus sociaux et politiques censés composer cette dimension partagée des projets éoliens. Les trajectoires individuelles qui accompagnent ces tensions montrent que les individus se construisent une posture d'opposition à mesure que les dispositifs politiques et institutionnels qu'ils sollicitent font défaut. Ce ne sont pas des opposants de principe et ils n'ont pas de position définie par rapport à l'éolien lorsque le projet émerge.

ressource énergétique n'a jamais été débattu, que ce soit au niveau européen ou français. D'autre part, l'abord implicite du vent par sa seule dimension physique, fluide et foisonnante ne permet pas de saisir les multiples faisceaux d'usages et d'attachements constitutifs des paysages avec lesquels interfère son exploitation comme ressource énergétique. Les tensions qui en résultent restent imparfaitement comprises.

Les trajectoires individuelles qui accompagnent ces tensions montrent que les individus De ce point de vue, les oppositions et les enjeux de paysage qui leur sont sous-jacents ne doivent pas être analysés comme des conservatismes : ils peuvent tout aussi bien être regardés comme une demande de prise en compte de la dimension collective de l'éolien et donc une contribution potentielle à l'émergence de nouvelles formes de citoyenneté énergétique. 


\section{BIBLIOGRAPHIE}

Briffaud, S., « Découverte et représentation d'un paysage : Les Pyrénées du regard à l'image (XVIIIXIX ${ }^{\mathrm{e}}$ siècles) », dans A. Roger (dir.), La Théorie du paysage en France (1974-1994), Seyssel, Champ Vallon, coll. « Pays/Paysages », 1995, p. 224-259.

Callon, M., « Civilizing markets : Carbon trading between in vitro and in vivo experiments », Accounting, Organizations and Society, vol. 34, $\mathrm{n}^{\circ}$ 3-4, 2009, p. 535-548.

Coriat, B., « Le retour des communs », Revue de la régulation, n 14, automne 2013.

Dewarrat, J.-P., Quincerot, R., Weil, M., Woeffray, B., Paysages ordinaires : de la protection au projet, Bruxelles, Mardaga, coll. « Architecture + recherches », 2003.

FR (2013) Loi n 2013-312 du 15 avril 2013 visant à préparer la transition vers un système énergétique sobre et portant diverses dispositions sur la tarification de l'eau et sur les éoliennes, JORF nº089 du 16 avril 2013 page 6208.

Labussière, O. et Nadaï, A., « Unexpected wind power 'potentials' : the art of planning with inherited socio-geographical configurations (France) », Scottish Geographical Journal, vol. 130, n 3, 2014, p. 152-167.

Latour, B., " Turning around Politics : A Note on Gerard de Vries' Paper », Social Studies of Science, vol. $37, \mathrm{n}^{\circ}$ 5, 2007, p. 811-820.

Le Baut-Ferrarese B. et Michallet, I., Traité de droit des énergies renouvelables, Paris, Éditions Le Moniteur, $2^{\mathrm{e}}$ édition, 2012.

Marres, N., «The Issues Deserve More Credit : Pragmatist Contributions to the Study of Public Involvement in Controversy ", Social Studies of Science, $\mathrm{n}^{\circ}$ 37, 2007.

Nadaï, A. «"Planning", "siting" and the local acceptance of wind power : Some lessons from the French case », Energy Policy, vol. 35, n 5, 2007a, p. 2715-2 726.

Nadaï, A., « Degré zéro : Portée et limites de la théorie de l'artialisation dans la perspective d'une politique du paysage », Cahiers de géographie du Québec, vol. 51, n 144, $2007 \mathrm{~b}$.

Nadaï, A. et Labussière, O., « Wind power planning in France (Aveyron) : from State regulation to local experimentation », Land Use Policy, vol. 26, n 3, 2009, p. 744-754.

Nadaï, A. et Labussière, O., « Birds, turbines and the making of wind power landscape in South France (Aude) », Landscape Research, vol. 35, n² 2, 2010, p. 209-233.

Nadaï, A., « Politique de l'énergie et paysages éoliens » dans Oueslati, W. (dir.), Analyses économiques du paysage, Versailles, Éditions Quae, 2011, p. 247-263.

Nadaï, A. et Labussière, O., «Eoliennes : États d'un paysage d'État », La Pierre d'Angle. Revue des Architectes des Bâtiments de France, Paris, n 56-57, 2011, p. 28-31.

Nadaï, A. et Labussière, O., « Wind power and the emergence of the Beauce landscape (Eure-etLoir, France) », Landscape Research, 2013, URL : http://www.tandfonline.com/doi/full/ 10.1080/01426397.2013.784732\#.VcnGXueolrQ.

Ostrom, E., Gouvernance des biens communs. Pour une nouvelle approche des ressources naturelles (1990), Bruxelles, De Boeck, 2013. 
Ostrom, E. et Basurto, X., « Façonner des outils d'analyse pour étudier le changement institutionnel », Revue de la régulation, $\mathrm{n}^{\circ}$ 14, automne 2013.

Trom D., « La production politique du paysage : éléments pour une interprétation des pratiques ordinaires de patrimonialisation de la nature en Allemagne et en France », thèse de doctorat sous la direction de Pierre Favre, Institut d'études politiques de Paris.

\section{NOTES}

1. Il s'agit d'un surnom donné au village en question à des fins de confidentialité.

2. La ZDE a été récemment annulée dans le cadre du débat parlementaire et du vote relatifs à la loi Brottes (FR, 2013).

3. Nous nous inspirons ici de Michel Callon (2009).

4. Entretien avec la Diren Île-de-France, le 22 février 2008.

5. Il s'agit là, comme pour Ventville, de surnoms donnés à des fins de confidentialité.

6. Entretien avec le SDAP 77, le 21 octobre 2008.

7. Entretien avec M. et Mme A., le 19 mars 2009.

8. Entretien avec M. et Mme A., le 19 mars 2009.

9. Le décret de mise en application des ZDE mentionne la Convention européenne du paysage (CEP) pour justifier de la nécessité de prendre en compte les paysages. S'agissant de la concertation, elle est demandée entre les services de l'État et les collectivités locales, alors qu'elle est «appréciée » (p. 20) s'agissant des citoyens. Tout en mentionnant la CEP, ce décret met l'accent sur la « valeur patrimoniale » des paysages. Il renvoie à une liste de documents types et d'« études paysagères reconnues " tels que PLU, Scot, Sage, chartes de PNR, schémas éoliens, monographies paysagères des CAUE, atlas départementaux de paysages comme pouvant aider à identifier ces paysages patrimoniaux.

10. Entretien avec M. et Mme A., le 19 mars 2009.

11. Entretien avec M. et Mme C., le 18 mars 2009.

12. Entretien avec le maire de Ventville, le 24 octobre 2008.

13. Entretien avec M. et Mme A., le 19 mars 2009.

14. Entretien avec le maire de Ventville, le 24 octobre 2008.

15. Entretien avec M. et Mme A., le 19 mars 2009.

16. Ibid.

17. Ibid.

18. Ibid.

19. Entretien avec le président de l'APPGS, le 22 octobre 2008.

20. Ibid.

21. Entretien avec M. et Mme A., le 19 mars 2009.

22. Entretien avec M. et Mme N., le 21 avril 2009.

23. Entretien avec le maire de Ventville, le 24 octobre 2008.

24. Entretien avec M. et Mme A., le 19 mars 2009.

25. Ibid.

26. Entretien avec M. et Mme C., le 18 mars 2009.

27. Entretien avec M. et Mme N., le 21 avril 2009.

28. Entretien avec le maire de Ventville, le 24 octobre 2008.

29. Préfecture de Seine-et-Marne. Commission départementale de la nature, des paysages et des sites. Permis de construire éoliens sur les communes de Ventville et de Soville. Compte rendu du 8 juillet 2008. 7 p.

30. Du 7 octobre au 7 novembre 2008. 
31. Entretien avec M. et Mme A., le 19 mars 2009.

32. Entretien avec M. et Mme C., le 18 mars 2009.

33. La notion d'" externalité compatible ", au sens d'un partage marché/non-marché compatible avec le redéploiement en cours (libéralisation) du secteur de l'électricité, a été proposée pour caractériser la dimension performative des paysages de l'énergie qui émergent avec difficulté de cet éolien de portage privé (Nadaï, 2011).

34. Cet acronyme signifie « Not in my backyard» (en français, «Pas dans mon jardin »). Il est utilisé pour qualifier l'attitude de personnes s'opposant à l'implantation, à proximité de chez eux, d'une infrastructure pourtant supposée reconnue d'intérêt public. Cette notion a donc un fort pouvoir de connotation incivique: tout en reconnaissant l'utilité de l'infrastructure, ces opposants tentent d'en éloigner les effets négatifs.

\section{RÉSUMÉS}

Cet article part d'un projet éolien contesté et conflictuel dans un village de Seine-et-Marne (France). Il suit l'émergence et la structuration de trajectoires d'opposants dans leur constitution progressive, en explorant les rôles et les devenirs opposés de communs paysagers. À la croisée de ces devenirs opposants et paysagers, l'analyse met en lumière les modalités selon lesquelles, en France, l'accompagnement institutionnel échoue à articuler des communs paysagers hétérogènes pour soutenir l'émergence de projets éoliens partagés.

This article starts with the opposition to a wind farm project which led to conflict in a village in Seine-et-Marne (France). It follows the emergence and the gradual structuring of the trajectories of the opposing parties by exploring the roles and different opposing projects for shared landscapes. At the intersection between these conflicting changes and projected landscapes, the analysis sheds light on the reasons why institutions in France fail to establish the connection between heterogeneous shared landscapes to support the emergence of joint wind farm projects.

\section{INDEX}

Keywords : wind energy, shared landscapes, landscape, issue, public

Mots-clés : éolien, communs, paysage, enjeu, public

\section{AUTEURS}

\section{ALAIN NADAÏ}

Alain Nadaï est socioéconomiste au Centre international de recherche sur l'environnement et le développement. Ses recherches portent sur les processus de transition énergétique à différentes échelles et selon différentes perspectives : paysages de l'énergie, nouvelles technologies de l'énergie (NTE), politiques climat-énergie et les communautés énergétiques durables. nadai[at]centre-cired[dot]fr 


\section{OLIVIER LABUSSIĖRE}

Olivier Labussière est maître de conférences en géographie et aménagement, Institut de Géographie Alpine. Ses recherches portent principalement sur les mutations des savoirs, les pratiques et méthodes de l'aménagement et la recomposition des paysages et territoires en contexte de transition énergétique.

olivier.labussiere[at]ujf-grenoble[dot]fr

http://www.pacte-grenoble.fr/blog/membres/labussiere-olivier/ 